\title{
ORIGINAL \\ ETIOLOGICAL GENDER DIFFERENCES IN PSYCHIATRIC DISORDERS
}

PROF-1163

\author{
DR. IMTIAZ AHMAD DOGAR \\ MBBS, MCPS (Psych) FCPS (Psych) \\ Head Department of Psychiatry \\ \& Behavioral Sciences, \\ Punjab Medical College Faisalabad, Pakistan \\ MISS. IRAM HINA \\ Psychologist, MSc. Applied Psychology
}

\author{
MISS. MEMOONA KIRAN \\ Psychologist, MSc. Applied Psychology \\ Miss. Qurat-ul-ain Arshed \\ Psychologist, MSc. Applied Psychology \\ Miss. Raisa Nazir. \\ Psychologist, MSc. Applied Psychology
}

\begin{abstract}
Background: Recent studies have shown particular differences found in both gender neurotic disorders but not in psychotic disorders with regard to environmental factors. But research in this area in Pakistan is direly needed. Aims \& Objectives: To find out what gender; either male or female more affect from environmental factors whether they are susceptible of that disorder or not and to find out the "etiology of gender differences in psychological disorder". Design: Non-interventional comparative study. Setting: Department of Psychiatry, District Headquarters Hospital, Faisalabad. Period: From May 2005 to Oct 2005. Material and Methods: A total of 80 patients of all ages are taken from in which we divide the sample i.e. each disorder comprises of 20 patients 10 male and 10 females. The Hospital that used is DHQ Hospital Faisalabad. We have used the random selection. The study is comprises of patient having Mood disorder, Anxiety, schizophrenia and personality disorder. For the simple analysis of data, percentages were applied for the statistical analysis. Results: The result showed that hypothesis is supported because there are significant differences (i.e. $42.5 \%>30 \%$ ) found as environmental factors affect more universally on females. Conclusion: Environmental factors affect more in women psychological health. Etiological gender differences in psychological disorders with special reference of mood disorder, anxiety disorder, schizophrenia and personality disorders are real and these gender differences may be due to their both nature or nurture gender. There are significant etiological gender differences in neurotic disorders i.e. Depression and anxiety disorder and minor difference in psychotic disorders i.e. Schizophrenia and mania.
\end{abstract}

\section{INTRODUCTION}

Gender identity is "A continuous and persistent sense of ourselves as male or female". Boys and girls are biologically different. It would be surprising if it were not so. Equally, it would be hard to argue that we do not treat them differently, and have different expectations of their behavior. What might not be appreciated are the subtleties of such expectations ${ }^{1}$.

Gender is a critical determinant of mental health and 
mental illness. The morbidity associated with mental illness has received substantially more attention than the gender specific determinants and mechanisms that promote and protect mental health and foster resilience to stress and adversity.

Gender determines the differential power and control men and women have over the socioeconomic determinants of their mental health and lives, their social position, status and treatment in society and their susceptibility and exposure to specific mental health risks.

Gender differences occur particularly in the rates of common mental disorders - depression, anxiety and somatic complaints. These disorders, in which women predominate, affect approximately 1 in 3 people in the community and constitute a serious public health problem $^{2}$.

Unipolar depression, predicted to be the second leading cause of global disability burden by 2020 , is twice as common in women. Depression is not only the most common women's mental health problem but may be more persistent in women than men. More research is needed. Many cases of depression are triggered by stressful life events, yet not everyone becomes depressed under such circumstances. The intensity and duration of these events, as well as each individual's genetic endowment, coping skills and reaction, and social support network contribute to the likelihood of depression. That is why depression and many other mental disorders are broadly described as the product of a complex interaction between biological and psychosocial factors. In the United States, approximately $20-25 \%$ of women and $12 \%$ of men will experience a serious depression at least once in their lifetimes. Among children, depression appears to occur in equal numbers of girls and boys. However, as girls reach adolescence, they tend to become more depressed than boys do. This gender difference continues into older age.

Women may be more likely than men to seek treatment. They may be more willing to accept that they have emotional symptoms of depressed mood and feelings of worthlessness or hopelessness. Men may be less willing to acknowledge their emotional symptoms and more apt to suppress their depression through the use of alcohol or other substances. In such cases depression can be "masked," or viewed only as alcohol or drug dependency/abuse rather than as clinical depression. Women may be more prone to depression because of the possible effects of hormones. Women have frequent changes in their hormone levels, from their monthly menstrual cycles, to the time during and after pregnancy, to menopause. Some women develop a depressive illness around these events.

Gender differences in bipolar affective disorder have also been described. Thus, women may have a higher risk of developing rapid-cycling bipolar disorder and dysphoric or mixed mania. Furthermore, women may be more likely to present with an initial depressive episode before onset of mania and to suffer from a greater number of depressive episodes and from longer, more refractory episodes throughout the course of their illness. However, whether there are gender differences in incidence or age at onset of bipolar disorder remains unclear.

One study ${ }^{3}$ has shown that stressful life events that may differentially affect women, particularly pregnancy and the puerperium have been associated with onset of mania. Similarly possible psychosocial factors such as unemployment or abnormal work or social functioning were not associated with either gender or age at onset of bipolar disorder in this study. However, the influence of other social factors, which may particularly affect young men, including urban living, social deprivation, and alcohol or illicit drug abuse, cannot be discounted.

There are no marked gender differences in the rates of severe mental disorders like schizophrenia and bipolar disorder that affect less than $2 \%$ of the population. The lifetime prevalence of schizophrenia is commonly given at 1\%; however, a recent review of studies from around the world estimated it to be $0.55 \%$. The incidence of schizophrenia was given as a range of between 7.5 and 16.3 cases per 100,000 of the population. 
Gender differences have been reported in age of onset of symptoms, frequency of psychotic symptoms, course of these disorders, social adjustment and long term outcome. The disability associated with mental illness falls most heavily on those who experience three or more comorbid disorders. Again, women predominate.

Depression, anxiety, somatic symptoms and high rates of comorbidity are significantly related to interconnected and co occurrent risk factors such as gender based roles, stressors and negative life experiences and events that disproportionately affect women include gender based violence, socioeconomic disadvantage, low income and income inequality, low or subordinate social status and rank and unremitting responsibility for the care of others.

The high prevalence of sexual violence to which women are exposed and the correspondingly high rate of Post Traumatic Stress Disorder (PTSD) following such violence, renders women the largest single group of people affected by this disorder.

Economic and social policies that cause sudden, disruptive and severe changes to income, employment and social capital that cannot be controlled or avoided, significantly increase gender inequality and the rate of common mental disorders.

Gender bias occurs in the treatment of psychological disorders. Doctors are more likely to diagnose depression in women compared with men, even when they have similar scores on standardized measures of depression or present with indentical symptoms. Female gender is a significant predictor of being prescribed mood altering psychotropic drugs.

Violence related mental health problems are also poorly identified. Women are reluctant to disclose a history of violent victimization unless physicians ask about it directly. The complexity of violence related health outcomes increases when victimization is undetected and results in high and costly rates of utilization of the health and mental health care system.
In Pakistan there have been only few studies on etiological gender differences in Psychological disorders. In particular environmental factors and psychological disorders have been rarely addressed. The present study aims to find out what gender; either male or female more affect from environmental factors whether they are susceptible of that disorder or not and to find out the "etiology of gender differences in psychological health".

\section{MATERIAL AND METHODS}

The study is conducted to find "Etiological gender differences in psychiatric disorders" (EGDPD). A sample of 80 diagnosed patients of four disorders according to DSM IV, i.e. mood disorders, anxiety disorders, schizophrenia \& personality disorders (40 male, 40 female) of all ages were taken as the research subjects with the help of consultant psychiatrist through the technique of probability random sampling for selecting the sample.

\section{INSTRUMENTS}

DSM IV Axis I \& I|

The data was analyzed with the help of percentages.

\section{RESULTS}

This research is conducted for the purpose to know the etiological gender differences in psychological disorders. Research is conducted on four disorders i.e. Mood Disorder, Anxiety Disorder, Schizophrenia and Personality Disorders. To analyze the results first of all four tables of four disorders are arranged. The data is divided into 5 spectrums. Each scale is subdivided into II groups i.e. male and female. In $1^{\text {st }}$ spectrum the sample who effect from the environmental factors more or less $90 \%$ fall. In $2^{\text {nd }}$ one the sample who has approx. $70 \%$ environmental and $30 \%$ biological factors included. In $3^{\text {rd }}$ group which is labeled "mixed" contains which have mixed emerged causes of their disorder that is around $44 \%$ environmental and $55 \%$ biological problems. In $4^{\text {th }}$ group which is headed by "bio/en" the sample fall who has approx. $70 \%$ biological problems and 30\% environmental and in the last one "bio" sample fall who have about $90 \%$ biological problems. $\ln 5^{\text {th }}$ table the total no of the sample is obtained to know the "etiological 
gender differences in psychological disorders". In $6^{\text {th }}$ table the percentage is obtained.

\begin{tabular}{|c|c|c|c|c|c|c|c|c|c|}
\hline \multicolumn{10}{|c|}{ Table-I. Mood Disorder } \\
\hline \multicolumn{2}{|l|}{ En. } & \multicolumn{2}{|c|}{ En/Bio } & \multicolumn{2}{|c|}{ Mixed } & \multicolumn{2}{|c|}{ Bio/En } & \multicolumn{2}{|c|}{ Bio } \\
\hline Male & Female & Male & Female & Male & Female & Male & Female & Male & Female \\
\hline $90 \%$ & $90 \%$ & $70 / 30 \%$ & $70 / 30$ & $44 / 55 \%$ & $44 / 55 \%$ & $70 / 30 \%$ & $70 / 30 \%$ & - & $90 \%$ \\
\hline $90 \%$ & $90 \%$ & - & $70 / 30$ & $44 / 55 \%$ & - & $70 / 30$ & - & - & - \\
\hline $90 \%$ & $90 \%$ & - & - & - & - & $70 / 30$ & - & - & - \\
\hline- & $90 \%$ & - & - & - & - & $70 / 30$ & - & - & - \\
\hline
\end{tabular}

The (Table I) indicates that environmental problems are experienced more in female as compare to male. $90 \%$ environmental causes are located in 30\%male and $40 \%$ female. $10 \%$ male and $20 \%$ female have 70 environmental $/ 30 \mathrm{bio} \%$ causes of their illness. Mixed merged causes are found in $20 \%$ male and $10 \%$ female. $40 \%$ male and $10 \%$ female have 70 bio/30en causes and only $10 \%$ female have $90 \%$ causes of their psychological problem and no male fall in this category.
The (Table II) indicates that female have more environmental problems as compared to male. $50 \%$ male and $70 \%$ female have $90 \%$ environmental related problems. $0 \%$ male and $10 \%$ female have $70 \mathrm{en} / 30 \mathrm{bio} \%$ causes of their illness. 10\% male and $0 \%$ female have mixed merged causes and $30 \%$ male and only $10 \%$ female have $90 \%$ causes of their psychological problem.

\begin{tabular}{|c|c|c|c|c|c|c|c|c|c|}
\hline \multicolumn{9}{|c|}{ Table-II. Anxiety Disorder } \\
\hline \multicolumn{2}{|c|}{ En. } & \multicolumn{2}{|c|}{ En/Bio } & \multicolumn{2}{|c|}{ Mixed } & \multicolumn{2}{c|}{ Bio/En } & \multicolumn{3}{c|}{ Bio } \\
\hline Male & Female & Male & Female & Male & Female & Male & Female & Male & Female \\
\hline $90 \%$ & $90 \%$ & - & $70 / 30$ & $44 / 55 \%$ & - & - & - & $90 \%$ & $90 \%$ \\
\hline $90 \%$ & $90 \%$ & - & - & $44 / 55 \%$ & - & - & - & $90 \%$ & - \\
\hline $90 \%$ & $90 \%$ & - & - & - & - & - & - & $90 \%$ & - \\
\hline $90 \%$ & $90 \%$ & - & - & - & - & - & - & - & - \\
\hline $90 \%$ & $90 \%$ & - & - & - & - & - & - & - & - \\
\hline- & $90 \%$ & - & - & - & - & - & - & - & - \\
\hline- & $90 \%$ & - & - & - & - & - & - & - & - \\
\hline
\end{tabular}

The (Table III) indicates that there is a no significant gender difference in the etiology of Schizophrenia. $0 \%$ male and $10 \%$ female have approximately $90 \%$ environmental caused of their illness. $10 \%$ male and
$10 \%$ female have $70 \mathrm{en} / 30 \mathrm{bio} \%$ root of their illness. $20 \%$ male and $20 \%$ female have diverse fused causes. $20 \%$ male and $10 \%$ female have 70 bio/30en causes and $50 \%$ male and $50 \%$ female have $90 \%$ causes of their 
psychological problem which indicate that schizophrenia

etiological gender differences. and other psychotic disorders have no significant

\begin{tabular}{|c|c|c|c|c|c|c|c|c|c|}
\hline \multicolumn{10}{|c|}{ Table-III. Schizophrenia } \\
\hline \multicolumn{2}{|l|}{ En. } & \multicolumn{2}{|c|}{ En/Bio } & \multicolumn{2}{|c|}{ Mixed } & \multicolumn{2}{|c|}{ Bio/En } & \multicolumn{2}{|c|}{ Bio } \\
\hline Male & Female & Male & Female & Male & Female & Male & Female & Male & Female \\
\hline- & $90 \%$ & $70 / 30 \%$ & $70 / 30$ & $44 / 55 \%$ & $44 / 55 \%$ & $70 / 30 \%$ & $70 / 30 \%$ & $90 \%$ & $90 \%$ \\
\hline- & - & - & - & $44 / 55 \%$ & $44 / 55 \%$ & $70 / 30 \%$ & - & $90 \%$ & $90 \%$ \\
\hline- & - & - & - & - & - & - & - & $90 \%$ & $90 \%$ \\
\hline- & - & - & - & - & - & - & - & $90 \%$ & $90 \%$ \\
\hline- & - & - & - & - & - & - & - & $90 \%$ & $90 \%$ \\
\hline
\end{tabular}

The (Table IV) also shows little gender differences in the etiology of personality disorders. $60 \%$ male and $40 \%$ female are suffered from $90 \%$ environmental stressors. $10 \%$ male and $30 \%$ female have $70 \mathrm{en} / 30 \mathrm{bio} \%$ causes of their illness. $0 \%$ male and $20 \%$ female have mixed combined causes. 20\%male and $10 \%$ female have 70 bio/30en causes and only $10 \%$ male have $90 \%$ origin of their psychological problem and no female fall in this category.

\begin{tabular}{|c|c|c|c|c|c|c|c|c|c|}
\hline \multicolumn{10}{|c|}{ Table-IV. Personality disorder } \\
\hline \multicolumn{2}{|c|}{ En. } & \multicolumn{2}{|c|}{ En/Bio } & \multicolumn{2}{|c|}{ Mixed } & \multicolumn{2}{|c|}{ Bio/En } & \multicolumn{2}{|c|}{ Bio } \\
\hline Male & Female & Male & Female & Male & Female & Male & Female & Male & Female \\
\hline $90 \%$ & $90 \%$ & $70 / 30 \%$ & $70 / 30 \%$ & - & $44 / 55 \%$ & $70 / 30 \%$ & $70 / 30 \%$ & $90 \%$ & - \\
\hline $90 \%$ & $90 \%$ & - & $70 / 30 \%$ & - & $44 / 55 \%$ & $70 / 30 \%$ & - & - & - \\
\hline $90 \%$ & $90 \%$ & - & $70 / 30 \%$ & - & - & - & - & - & - \\
\hline $90 \%$ & $90 \%$ & - & - & - & - & - & - & - & - \\
\hline $90 \%$ & - & - & - & - & - & - & - & - & - \\
\hline $90 \%$ & - & - & - & - & - & - & - & - & - \\
\hline
\end{tabular}

The (Table V) shows the total no. of patients of all disorders which are under observation of this research and table 6 shows the percentage of the results of table 5. $30 \%$ male(12 out of 80 patients) and $42.5 \%$ (17 patients out of 80 patients) affected from environmental stressors. $10 \%$ male (4 out of 80 patients) and $17.5 \%$ female (7 out of 80 patients) faced $70 \mathrm{en} / 30 \mathrm{bio} \%$ problems. $15 \%$ male ( 6 out of 80 patients) $15 \%$ female $(6$ out of 80 patients)faced mixed approximately $50 \%$ en/bio problems. 30\% male (12 out of 80 patients) and 7.5\% female (3 out of 80 patients) faced 70bio/30en $\%$ problems. $15 \%$ male (4 out of 80 patients) and $17.5 \%$ 
female (7 out of 80 patients) faced $90 \%$ biological problems.

\begin{tabular}{|c|c|c|c|c|c|c|}
\hline \multicolumn{7}{|c|}{ Table-V. Gender different found in Psychological disorder. } \\
\hline Gender & En. & En/Bio & Mixed & Bio/En & Bio & Total \\
\hline Male & 12 & 4 & 6 & 12 & 6 & 40 \\
\hline Female & 17 & 7 & 6 & 3 & 7 & 40 \\
\hline
\end{tabular}

\begin{tabular}{|c|c|c|c|c|c|c|}
\hline \multicolumn{7}{|c|}{ Table-VI. Percentage of gender difference found in Psychological disorder. } \\
\hline Gender & En. & En/Bio & Mixed & Bio/En & Bio & Total \\
\hline Male & $12 / 40 * 100=30 \%$ & $4 / 40 * 100=10 \%$ & $6 / 40 * 100=15 \%$ & $12 / 40 * 100=30 \%$ & $6 / 40 * 100=15 \%$ & 40 \\
\hline Female & $17 / 40 * 100=42.5 \%$ & $7 / 40 * 100=17.5 \%$ & $6 / 40 * 100=15 \%$ & $3 / 40 * 100=7.5 \%$ & $7 / 40 * 100=17.5 \%$ & 40 \\
\hline
\end{tabular}

\section{DISCUSSION}

The main objective of this study was to confirm the concept of etiological gender differences in Psychological disorders with special reference of Mood Disorder, Anxiety Disorder, Schizophrenia and Personality Disorders. Similar to the general findings in literature there was significant differences in neurotic disorders i.e. depression and anxiety disorder and minor difference in psychotic disorders i.e. schizophrenia and Mania. The results showed that hypothesis is supported b/c there are significant differences (i.e. $42.5 \%>30 \%$ ) found as environmental factors affect more universally on female. The reasons that affect on female are varied...... i.e. gender role....... the discriminatory behavior, she is sensitive by nature and the family expectations also made female more susceptible........ they are restricted to express their feelings $\mathrm{b} / \mathrm{c}$ of the societal pressure........stress are more in her life e.g. menstrual cycle, then the marriage , reproduction and domestic life menopause etc.

By examining the result of the mood disorder it shows that it is more frequently occur in females as they are more extremist in their emotions and take slight things on nerves like emotive problems. The environment in which the female lived makes her submissive, subordinate and compromising and the variety of the conflicting roles she has to perform in her life i.e. female $=$ daughter + daughter in law + wife + mother + mother in law etc. Whereas, the male does not have to leave his home after marriage and to go to the strange environment as female has to do. Female may be prone to depression $\mathrm{b} / \mathrm{c}$ of the possible effect of the hormones, and this affect cause emotional and behavioral problems. Females have the frequent changes in their hormones level from their monthly menstrual cycle to the time during and after the pregnancy to the menopause. Some women develop a depression around these event are the most probable cause of unipolar. In October 2000, the American Psychological Association convened a summit of more than 35 experts on women and depression, chaired by Carolyn Mazure PhD of Yale University. In April 2002, the APA released a 59 page report based on that summit, Summit on Women and Depression, according to the Summit Report: "Depressive symptoms and syndromes are associated with periods when gonadal hormones are undergoing considerable change, mainly during the premenstrual period, the postpartum period, and at the initiation of menopause." As many as 75 percent of women experience some premenstrual emotional and behavioral problems. Meanwhile, pregnancy and delivery produce dramatic changes in estrogen and progesterone levels, as well as major changes in the HPA axis, while perimenopause results in critical fluctuations in estrogen and other hormones. The Summit Report is quick to point out that these changes 
have not yet been definitively linked to mood disorders, though the onset of puberty when girls start experiencing depression in greater numbers than boys.

But in most of the cases I note that Mood disorders are not caused by the biological factors but is caused by the way as the gender response to the environment or their coping skills to the problems, as Nolen-Hoeksema ${ }^{4}$ has developed a theory of women's increased vulnerability to depression based on the identification of a self-focused coping style in response to depressed mood. In her research, she found relatively weak support for overall gender differences in personality characteristics of passivity and assertiveness, but she noted that differences occur in women's response to depression; they focus on negative emotions while men use distracting responses especially sports and athletic activities to cope with depression. During field work it has been noted that girls at adolescence are triggered by their greater exposure at this time to concerns with personal appearance, safety, and self-worth. Their a ruminative style is seen as providing a general link to women's tendency to have longer and more severe depressive episodes, specifically, rapid cycling and mixed-state bipolar forms. Nolan-Hoeksema ${ }^{5}$ found that physical activity has been found to enhance the self concept of women who are depressed. There is also evidence that women experience more life events than men. In bipolar disorder females have more depressive episode and more mixed episodes than men with the illness.

In anxiety disorders psychosocial factors may also play a role in the higher rates of anxiety in females. Several studies support the notion that having some "masculine" traits can protect against anxiety. It is plausible that an assertive, goal-oriented, competitive style may lead a person to be less avoidant and therefore less anxious, as says Katherine Shear, M.D., writing in Gender and Its Effects in Psychopathology ${ }^{6}$.

In schizophrenia, genetics plays a strong role in it. The onset can be delay but if the susceptibility is present then the person will definitely become schizophrenic. This study shows that affective symptoms are more in female and the psychotic symptoms are found in males.

In light of the differences in age of onset between men and women, it is tempting to speculate about the possible influence of gender on the height of the threshold for developing schizophrenia. There is some evidence that gendered behavior, not only genetic sex, can influence brain morphology ${ }^{7}$. If that is the case, it is certainly possible that the stress buffering that results from supportive families and protective social networks (more prevalent in young women than in young men) delays the onset of schizophrenia, promotes premorbid skill acquisition, and, consequently, assures a more favourable course of illness.

The brainchild of Dr. Mary Seeman, ${ }^{8}$ chair of schizophrenia studies at the University of Toronto, who believes it's time "physicians become aware of the fact that schizophrenia as they know it is a male model and that woman don't fit that standard picture." But in this study we didn't find it there is also as many women as men suffering from schizophrenia.

When it comes to personality disorder it is found in male excessively as the criteria support it and so as the reaction. Family environment is crucial in the development of a child's brain and personality. Genetics can only go so far, and environment works to shape a child's mind after the child has left a mother's womb. Jones $^{9}$ discussed how a financially unstable family and child abuse or neglect are associated with criminal behavior. Environment is important for a child to grow and develop into a normal, prospering adult. Without proper nurturance, guidance, and support, no child, male or female, will learn coping strategies, learn life skills, or grow up with a strong sense of right and wrong and respect other people. A woman is likely to label herself a "whore" and a male narcissist to self-style himself a "vicious, unrepentant criminal"10 Yet, these again are traditional social roles. Men are likely to emphasise intellect, power, aggression, money, or social status. Women are likely to emphasise body, looks, charm, sexuality, feminine "traits", homemaking, children and 
childrearing - even as they seek their masochistic punishment, as the findings of Eagly, A. H., Makhijani, M. G., \& Klonsky, B. G.

Results of this research conforms the fact that female is as a gender is susceptible, as we see in the result's biological factors are more in female than male (i.e. $17.5 \%>15 \%$ ). However more studies are needed in this area to further explore these gender differences in relation to psychiatric disorders.

\section{REFERENCES}

1. Nicholson J. (1984) Men and Women: How different are they? Oxford: Oxford University Press.

2. Gender and women's mental health. Gender disparities and mental health: The Facts. Available from URL: http://www.who.int/mental_health/prevention/genderwo men/en/

3. Noel Kennedy, M.D., M.R.C.Psych., Jane Boydell, M.D., M.R.C.Psych., Sri Kalidindi, M.D., M.R.C.Psych., Paul Fearon, M.D., M.R.C.Psych., Peter B. Jones, M.D., Ph.D., M.R.C.Psych., Jim van Os, M.D., Ph.D., M.R.C.Psych. and Robin M. Murray, M.D., D.Sc., F.R. Psych. Gender Differences in Incidence and Age at Onset of Mania and Bipolar Disorder Over a 35-Year Period in Camberwell, England Am J Psychiatry 162:257-262,
February 2005.

4. Nolen-Hoeksema S: Gender differences in coping with depression across the lifespan. Depression 3:81-90, 1995.

5. Nolen-Hoeksema S: Sex Differences in Depression. Palo Alto, Stanford University Press, 1990.

6. Stephanie Sampson, M.A, Being Female and Anxious Anxiety Disorders in Women. Anxiety Disorders Association of America. Katherine Shear, M.D. Ellen Frank, Ph.D. Gender and Its Effects in Psychopathology. American Psychiatric Press, 2000

7. Lewine RRJ, Seeman MV. Anatomy of difference-difference in anatomy. In: Seeman MV, editor. Gender and psychopathology. Washington (DC): American Psychiatric Association Press; 1995. p 131-58.

8. Kreidie A Schizophrenia affects the sexes differently. The Medical Post 2005 Oct 4

9. Lowenstein, L. F. (2003). The genetic aspects of criminality. Journal of Human Behavior in the Social Environment, 8, 63-78

10. Dr. V. Sam "Malignant Self Love Narcissism Revisited" $7^{\text {th }}$ edition 2006 Feb ISBN: 8023833847Publisher: Narcissus Publications 